\title{
Evaluation of Clinical and Pathological Features of Follicular Lymphoma - A Three Year Descriptive Study in a Regional Cancer Centre in Western India
}

\author{
Rukmini Bezbaruah ${ }^{1}$ and Biren Parikh ${ }^{2 *}$ \\ ${ }^{1}$ DM Oncopathology Resident, Department of Oncopathology, The Gujarat Cancer Research Institute, India \\ ${ }^{2}$ Associate Professor, Department of Oncopathology, The Gujarat Cancer Research Institute, India
}

*Corresponding author: Biren Parikh, Associate professor, Department of Oncopathology, The Gujarat Cancer Research Institute, India

\begin{abstract}
Background: Follicular lymphoma (FL) is the most common "indolent" lymphoma, occurring with a frequency of approximately $2 / 100,000$ in the Western world [1]. There are only a few studies about the epidemiological and geographic trends of follicular lymphoma in India.

Aims and objectives: (i) To analyze the demographic and clinicopathologic characteristics of FL, (ii) To study the histopathological features of FL.

Materials and methods: This was a retrospective study where cases of FL were collected from the department of Oncopathology, at a tertiary care centre over 3 years from January 2017 to December 2019. Statistical analysis was done using the Software Statistical Package for the Social Sciences (SPSS) Version 20.

Results: Age range was from 35 to 84 years. Out of 69 cases, 18 cases $(26 \%)$ were grade I, 32 cases $(46 \%)$ were grade II, and 19 cases (27\%) were grade III. Ki67 showed significant correlation in case of grade, stage, FLIPI score, number of lymph node areas and bone marrow involvement $(P<0.05)$. There were three cases of FL showing transformation to Diffuse Large B Cell Lymphoma. Serum lactate dehydrogenase (LDH) is a non-specific marker for lymphoma whose prognostic significance is well established for both indolent and aggressive lymphomas at the time of diagnosis.

Conclusion: This study highlights the various clinical, pathological, and histopathological features of FL. Features like grade, stage, the impact of age, nodal areas, bone marrow, and peripheral blood involvement by FL were highlighted.
\end{abstract}

\section{Keywords}

Follicular lymphoma, FLIPI, Ki67

\section{Introduction}

Follicular lymphoma (FL) is the most common of the so-called "indolent" lymphomas, occurring with a frequency of approximately $2 / 100,000$ in the Western world [1]. It arises from a complex interaction of genetic and environmental factors, though most patients do not have clear risk factors at presentation. In India, it is the second most common B-Non-Hodgkin Lymphoma (NHL) subtype after diffuse large B-cell lymphoma (DLBCL). There are only a few studies about the epidemiological and geographic trends of FL in India.

Follicular lymphoma is characterized by the reciprocal translocation $\mathrm{t}(14 ; 18)(\mathrm{q} 32 ; \mathrm{q} 21)$, which is present in 85 to $90 \%$ of cases [2]. It is the first step in the genesis of lymphoma, occurring within the bone marrow during B-cell lymphopoiesis [1]. This is the likely initial necessary although not sufficient abnormality present early during a multihit pathway which ultimately leads to the development of clinically overt lymphoma. Treatment options for patients with FL vary based on the stage of the disease, age of patient, performance status, comorbidities, and therapeutic goals.

The natural history of FL is characterized by recurrent relapses and progressively shorter remissions with a median survival of 10 years. However, it is necessary to identify the high-risk cases as they require intensive treatment.

Citation: Bezbaruah R, Parikh B (2020) Evaluation of Clinical and Pathological Features of Follicular Lymphoma - A Three Year Descriptive Study in a Regional Cancer Centre in Western India. Int J Oncol Res 3:025. doi.org/10.23937/2643-4563/1710025

Accepted: November 20, 2020: Published: November 22, 2020

Copyright: (C) 2020 Bezbaruah R, et al. This is an open-access article distributed under the terms of the Creative Commons Attribution License, which permits unrestricted use, distribution, and reproduction in any medium, provided the original author and source are credited. 
A lot of progress has been made in the treatment of FL but still, a significant portion of patients have a poor outcome. During the past decades, several potential prognostic factors and risk models in patients with FL were studied. This was to identify patients at risk for poor outcome. But only the Follicular Lymphoma International Prognostic Index (FLIPI), which was established in 2004, was widely used to predict overall survival. The (FLIPI) classified patients with FL into three groups according to overall survival (OS). Five adverse prognostic factors were considered: 1) Age 2) Ann Arbor stage 3) Hemoglobin level 4) Number of nodal areas and 5) LDH level-leading to the definition of three risk groups related to OS [1]. The use of Rituximab has substantially improved the outcome of patients.

The objective of this study was: i) To analyze the demographic and clinicopathologic characteristics of FL ii) To study the histopathological features of FL.

\section{Materials and Methods}

\section{Case selection}

This was a retrospective observational study where cases of FL were collected from the department of Oncopathology, at a tertiary care centre over 3 years from January 2017 to December 2019. A total of 69 cases of FL were retrieved out of the total NHL cases diagnosed. Age, sex, presenting complaints, Complete blood count (CBC), Lactate dehydrogenase (LDH), histological findings with grade, proliferative index (Ki67), bone marrow aspiration and biopsy findings, peripheral blood involvement were noted. The radiological examination included chest radiograph, Computed Tomography (CT), and ultrasonography of the abdomen. Radiological findings were collected from the case files and the institute's computer registry. The disease was staged according to Ann Arbor staging. Grading was done using the World Health Organisation (WHO) 2016 criteria [3]. Ethical clearance was obtained from the Institutional Review Board.

\section{Histopathology and Immunohistochemistry (IHC) of primary specimen and biopsy}

Samples consisted of excisional, incisional and trucut biopsies which were formalin-fixed, paraffin-embedded and cut into four to five-micron sections and stained with hematoxylin and eosin $(\mathrm{H}$ and $\mathrm{E})$ and cases were diagnosed provisionally as NHL on light microscopy. For sub typing and final diagnosis, IHC examination was performed using a Ventana Benchmark Ultra machine automated staining system. Immunohistochemistry aided in confirmation of morphological diagnosis and particularly helped in differentiating follicular lymphoma from follicular hyperplasia and other low grade lymphoma like marginal zone lymphoma. A panel of antibodies were selected for this purpose both leukocyte and non-leukocyte markers. In cases of extranodal presentations and metastasis non-leukocyte markers were utilised for diagnosis. In the evaluation of the following IHC markers, the results were considered as either positive or negative in the tumor cells: CD3, CD10, CD20, CD23, BCL2, $\mathrm{BCL6}$, kappa and lambda immunoglobulin light chains, and MUM1/IRF4. CD21 and CD23 helped to identify the follicular architecture, especially in small biopsies, and highlight follicular dendritic cell meshworks which are often more irregular than those seen in normal follicles. Grading of $F L$ was done by counting the number of centroblasts in 10 representative neoplastic follicles, expressed per $40 \times$ objective high power field (hpf). Grade $1=0-5$ centroblasts $/ \mathrm{hpf}$; grade $2=6-15$ centroblasts/ hpf; grade $3=>15$ centroblasts/hpf. In grading of follicular lymphoma, IHC played a role. KI 67 proliferative index correlates with histological grading. The Ki-67 proliferative index was evaluated using the monoclonal MIB-1 antibody and assigned a percentage value that was calculated by scoring 500 tumor cell nuclei.

\section{Peripheral blood, bone marrow aspiration and tre- phine biopsies}

The bone marrow or peripheral blood was collected in ethylenediaminetetraacetic acid (EDTA) vacutainer. A morphological evaluation was done from the Wrightstained peripheral smears and bone marrow aspirates. All the samples were processed within 24 hours. If the bone marrow aspiration and peripheral smear showed involvement by lymphoma confirmation was done by immunophenotyping. Following panel was used (Table 1).

Table 1: Panel of antibodies used in immunophenotyping for Chronic Lymphoproliferative disorder (CLPD).

\begin{tabular}{|l|l|l|l|l|}
\hline SI no. & Fluorochrome & $\begin{array}{l}\text { B-CLPD 1 surface } \\
\text { Tube 1 }\end{array}$ & $\begin{array}{l}\text { B- CLPD 2 surface } \\
\text { Tube 2 }\end{array}$ & $\begin{array}{l}\text { B-CLPD 2 surface } \\
\text { Tube 3 }\end{array}$ \\
\hline 1 & V450 & CD20 & & CD45 \\
\hline 2 & V500 & CD45 & Lamda & CD25 \\
\hline 3 & FITC & CD23 & Kappa & CD103 \\
\hline 4 & PE & CD200 & CD5 & CD11C \\
\hline 5 & Per CP Cy5.5 & CD79b & CD19 & CD19 \\
\hline 6 & PECy7 & CD19 & & \\
\hline 7 & APC & CD10 & & \\
\hline
\end{tabular}




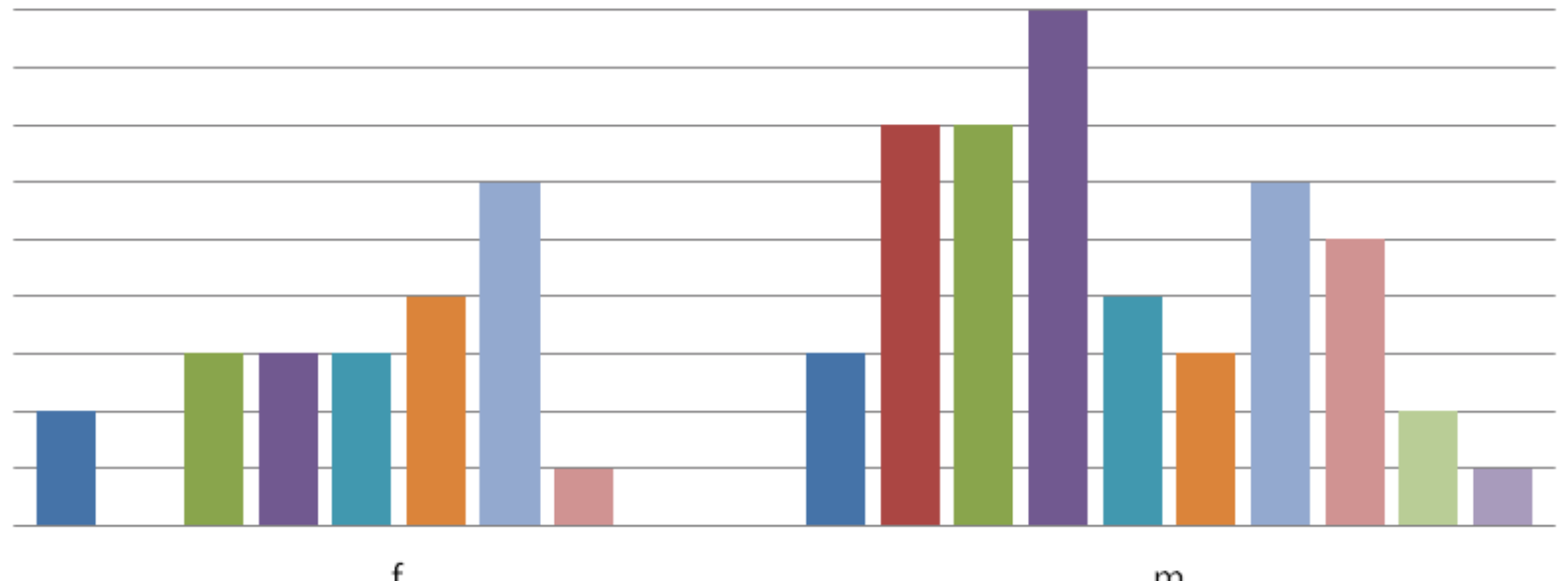

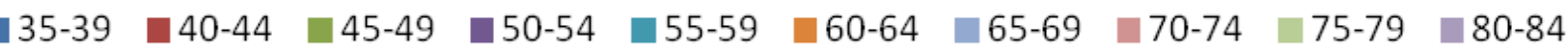

Figure 1: Distribution of cases in both sexes.

Trephine biopsies which showed involvement by lymphoma on morphology were also subjected to IHC for confirmation.

\section{Prognostic scoring}

FLIPI score was counted based on the following - Age $>60$ years, $\geq 4$ nodal sites of disease, Ann Arbor Stage III-IV, Hemoglobin level < $12 \mathrm{~g} / \mathrm{dl}$, LDH level > upper normal reference level (1 point for each variable present). Based on this, cases were stratified into three risk groups - Low risk (0 or 1), Intermediate risk (2), High risk (3-5).

\section{Statistical analysis}

Statistical analysis was done using the Software Statistical Package for the Social Sciences (IBM SPSS statistics version 20 IBM Corp, Armonk, NY). The qualitative data were presented in frequencies and percentages, and quantitative data were presented by mean or median. The Chi-square test was used to compare the patient characteristics. P-value $\leq 0.05$ was considered to be statistically significant.

\section{Results}

\section{Demographic characteristics}

There were 69 patients diagnosed as FL in three years from 2017 to 2019, out of all cases of NHL. Age range was from (35 to 84 ) years. Out of these, there were 47 men $(\mathrm{m})$ and 22 women $(\mathrm{f})$ (men:women $=2: 1$ ) with a median age of $55 \pm 11.62$ years, mean age was 56.04 years. Most of the cases were in the age group (50-54) years and (65-69) years. In men, commonest age group was (50-54) years. In women, the most number of cases were diagnosed in (65-69) years, age group (Figure 1). Mean White blood count (WBC) was $14.0 \pm 20.87$ $\times 10^{9} / \mathrm{L}$. Lactate dehydrogenase was elevated in 62 cas- es. The most common nodal anatomic site was the cervical lymph nodes (29\%) followed by the inguinal nodes (24.6\%) (Figure 2). Almost all the cases presented with lymphadenopathy. Nodal involvement was noted in 67 cases and extranodal involvement was noted in 2 cases. One was a case of FL in the breast in a 69-years-old woman. Another was a case of $\mathrm{FL}$ in the parotid gland. Staging was done in all cases of FL and out of 69 cases, the highest number of cases were in stage III (44.9\%) followed by stage IV (34.8\%), stage II (18.8\%) and stage I (1.4\%).

\section{Histopathology}

All the cases had effacement of lymph node by follicular architecture with follicles of similar size present in the cortex and medulla. The percentage of centroblasts varied according to the grade. Grading was done according to the World Health Organization Classification of lymphoid neoplasm [4]. Out of 69 cases, 18 cases $(26 \%)$ were grade I, 32 cases (46\%) were grade II, 19 cases $(27 \%)$ were grade III. Two cases were diagnosed as FL with focal areas showing transformation to DLB$\mathrm{CL}$. There were 2 cases of $\mathrm{FL}$ showing transformation to B Cell NHL high grade. One case of collision tumour was diagnosed in a 57-year woman having synchronous metastatic serous papillary carcinoma and follicular lymphoma in pelvic node (Figure 3). Two cases had both follicular and diffuse pattern out of which one was grade II and other was grade III.

\section{Immunohistochemistry}

A panel of antibodies was used for diagnosis. All cases were positive for CD20, CD10 and Bcl-2. Sixty cases were positive for $\mathrm{BCl}-6$ and 6 cases were negative and in 3 cases Bcl-6 was not done. CD23 was positive in follicular dendritic cells in 15 cases. Proliferative index (Ki67) was performed in all 69 cases (Table 2). 


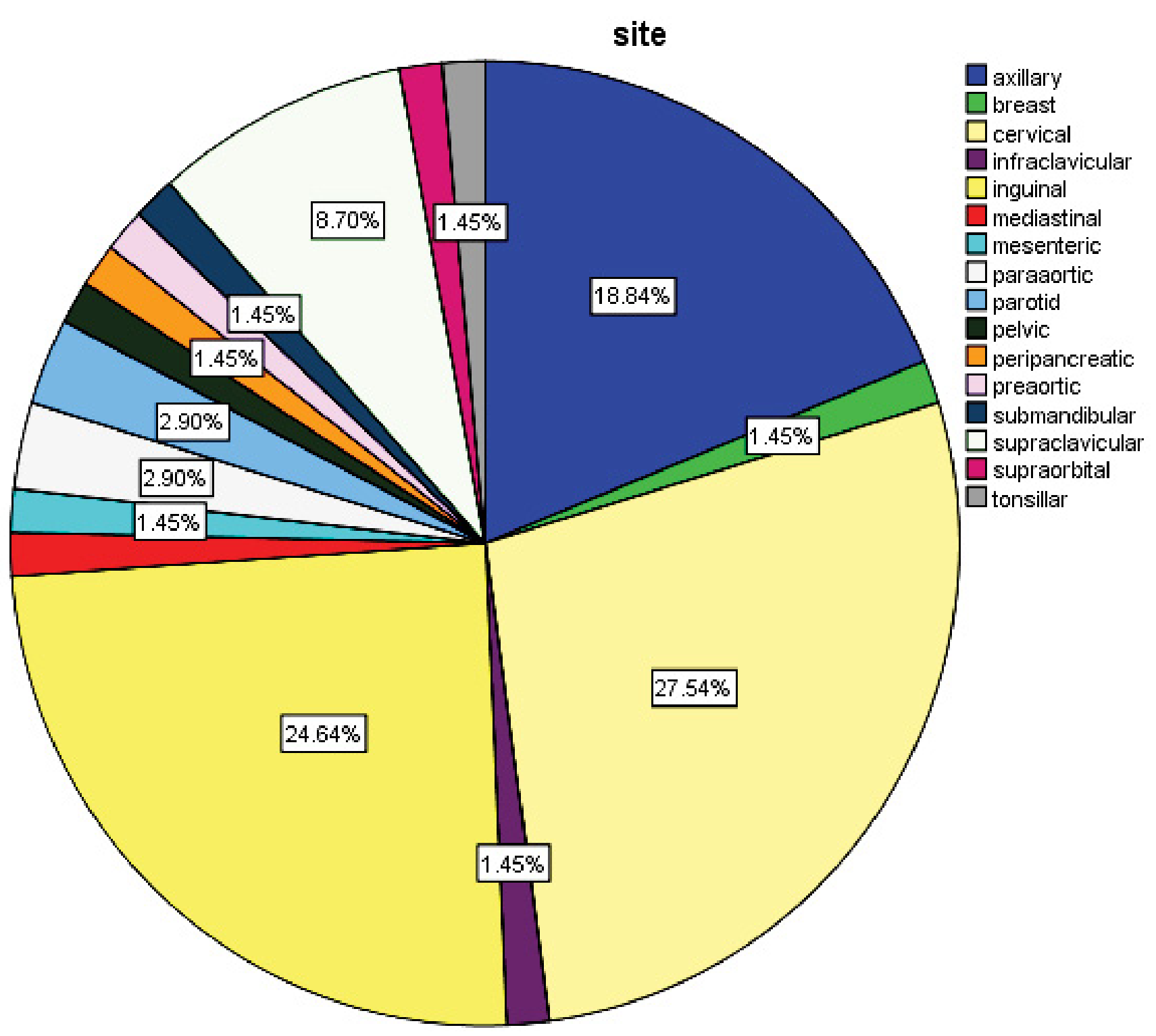

Figure 2: Distribution of cases according to various sites.

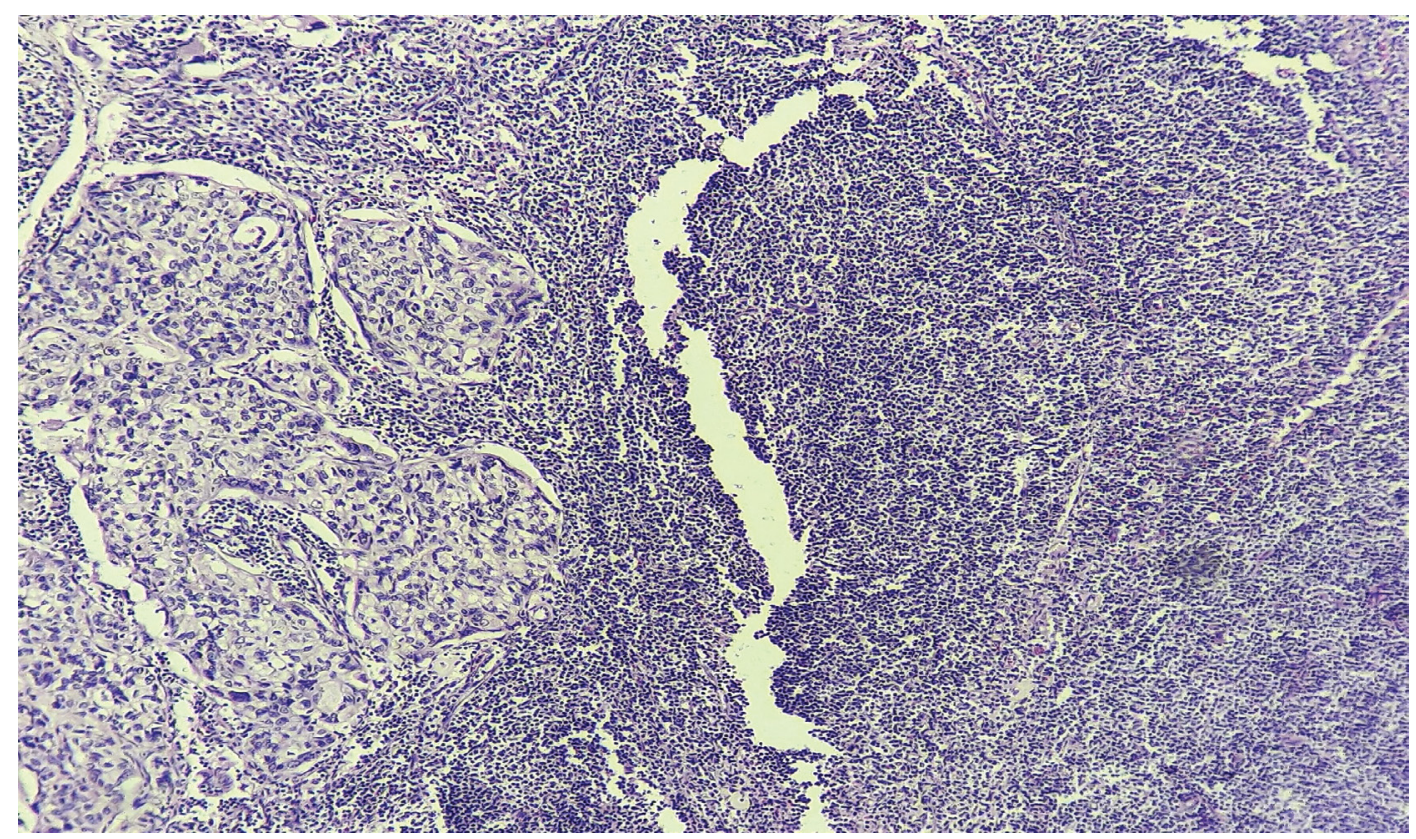

Figure 3: $(\mathrm{H}$ and $\mathrm{E}, \times 100)$ of pelvic node showing synchronous presence of metastatic adenocarcinoma and follicular lymphoma. 
Table 2: Proliferative index (Ki67) at diagnosis.

\begin{tabular}{|l|l|}
\hline Parameters (Ki67) & No. of Cases (\%) \\
\hline$<\mathbf{2 0 \%}$ & $22(31.9 \%)$ \\
\hline $\mathbf{( 2 1 - 4 0 )} \%$ & $27(39.1 \%)$ \\
\hline $\mathbf{( 4 1 - 6 0 )} \%$ & $16(23.2 \%)$ \\
\hline $\boldsymbol{\varnothing} \mathbf{6 1} \%$ & $4(5.8 \%)$ \\
\hline
\end{tabular}

Table 3: Patient characteristics.

\begin{tabular}{|c|c|c|}
\hline \multicolumn{2}{|l|}{ Characteristics } & \multirow{2}{*}{$\begin{array}{l}\text { Frequencies } \\
42(60.9 \%)\end{array}$} \\
\hline Age (years) & $\leq 60$ & \\
\hline & $>60$ & $27(39.1 \%)$ \\
\hline \multirow[t]{2}{*}{ LDH elevated } & No & $7(10.1 \%)$ \\
\hline & Yes & $62(89.9 \%)$ \\
\hline \multirow[t]{2}{*}{ Hemoglobin } & $\geq 12 \mathrm{~g} / \mathrm{dL}$ & $27(39.1 \%)$ \\
\hline & $<12 \mathrm{~g} / \mathrm{dL}$ & $42(60.9 \%)$ \\
\hline \multirow[t]{4}{*}{ Ann Arbor Stage } & I & $1(1.4 \%)$ \\
\hline & II & $13(18.8 \%)$ \\
\hline & III & $31(44.9 \%)$ \\
\hline & IV & $24(34.8 \%)$ \\
\hline \multirow[t]{2}{*}{ No. of nodal areas } & $\leq 4$ & $32(46.4 \%)$ \\
\hline & $>4$ & $37(53.6 \%)$ \\
\hline \multicolumn{2}{|l|}{ Extranodal involvement } & $2(2.9 \%)$ \\
\hline \multirow{3}{*}{ Grade } & 1 & $18(26.08 \%)$ \\
\hline & 2 & $32(46.4 \%)$ \\
\hline & $3 A+3 B$ & $19(27.5 \%)$ \\
\hline \multirow[t]{3}{*}{ FLIPI Risk groups } & I & $7(10.1 \%)$ \\
\hline & II & $12(17.4 \%)$ \\
\hline & III & $50(72.5 \%)$ \\
\hline \multirow[t]{2}{*}{ BM } & Yes & $13 / 69(18.8 \%)$ \\
\hline & No & $53 / 69(76.8 \%)$ \\
\hline \multirow[t]{2}{*}{$\mathrm{BX}$} & Yes & $21 / 69(30.4 \%)$ \\
\hline & No & $46 / 69(66.7 \%)$ \\
\hline \multirow{2}{*}{$\begin{array}{l}\text { Circulating lymphoma } \\
\text { cells }\end{array}$} & Yes & $7 / 69(10.1 \%)$ \\
\hline & No & $62 / 69(89.9 \%)$ \\
\hline
\end{tabular}

BX: Bone marrow biopsy; BM: Bone marrow aspiration

\section{Bone marrow involvement}

Bone marrow was available in 66 cases (95.6\%). Thirteen cases (18.8\%) showed involvement in both bone marrow aspiration and trephine biopsy but 8 cases $(11.6 \%)$ showed involvement only in bone marrow biopsy and aspiration was reported as uninvolved by lymphoma. In trephine biopsy, paratrabecular involvement by FL was seen in 15 cases $(21.7 \%), 3$ cases $(4.3 \%)$ showed diffuse pattern and 3 cases $(4.3 \%)$ showed nodal and interstitial involvement. Peripheral blood involvement by FL was seen in 7 cases (10.14\%).

Follicular Lymphoma International Prognostic Index score was calculated in all cases. Seven cases (10.1\%), were in the low-risk category, 12 cases (17.4\%) were in the intermediate-risk category and 50 cases
(72.5\%) were in the high-risk category (Table 3).

\section{Discussion}

Follicular lymphoma is a systemic neoplasm of the lymphoid tissue displaying germinal centre (GC) B cell differentiation. It represents $\sim 5 \%$ of all haematological neoplasms and $\sim 20-25 \%$ of all new non-Hodgkin lymphoma diagnoses in western countries [5]. Precise data from India is sparse [6]. In this study, we analysed the demographic and clinicopathologic characteristics of $\mathrm{FL}$ and its prognostic factors.

\section{Demographic characteristics}

The median age of patients in our study was 55 years ranging from 35 years to 84 years which is similar to Ajay Gogia, et al. [6] which had a median age of 51 years. Mean age was 56.04 years. Sylvia, et al. [7] had a mean age of 50 years. While Duarte, et al. [8] found a lower mean age at 35 years. Literature quotes the median age of follicular lymphoma to be a decade higher (5960) years in the developed countries [7]. Men to women ratio was 2:1 which is similar to Shih-Sung Chuang, et al. [9] which had a ratio 1.9:1. But Western studies have documented predominance in women [10]. Age $>60$ years at diagnosis is a risk factor in both scores FLIPI and FLIPI2 score [11]. The most common site of involvement was cervical lymph nodes (29\%) which are similar to Duarte, et al. [8].

Follicular Lymphoma is an indolent disease and presents in disseminated stage [10]. In our study, maximum cases were in stage III and IV which is similar to Sylvia, et al. [7] Serum lactate dehydrogenase is a non-specific marker for lymphoma whose prognostic significance is well established for both indolent and aggressive lymphomas at the time of diagnosis [12]. Lactate dehyrogenase was elevated in 62 out of 69 cases (89.8\%). There was a significant correlation of LDH with FLIPI score and risk groups $(P<0.05)$.

\section{Pathological features}

Histopathology along with IHC is the basis of the diagnosis of $\mathrm{FL}$ although it can also be based on the results of flow cytometry and cytogenetic analysis, if necessary. Histologically this neoplasm is composed of germinal centre $B$ cells typically centrocytes and centroblasts [4]. Low-grade B-cell lymphomas that are composed of nodular structures should be differentiated from follicular lymphoma. They are marginal zone B-cell lymphoma, mantle cell lymphoma (MCL) and chronic lymphocytic leukemia/small lymphocytic lymphoma. Tumor cells of marginal zone B-cell lymphoma proliferate in marginal zone areas and sometimes have follicular colonization. They often show differentiation to monocytoid B cells or plasma cells. Immunostaining of CD10 is useful to recognize regressed germinal centers. In follicular lymphoma cases, tumor follicles show stronger CD10 immunostaining. 
MCL is usually differentiated by cyclin D1 positivity on immunostaining. From a histological perspective, MCL shows monotonous medium-sized cell proliferation, pink macrophages and atrophic germinal centers. Chronic lymphocytic leukemia/small lymphocytic lymphoma forms nodular structures composed of small to medium-sized tumor cells, and within them, proliferation centers composed of prolymphocytes and paraimmunoblasts are seen. Tumor cells are positive for CD5 and $\mathrm{CD} 23$ by immunostaining.

The distinction of neoplastic follicles from benign follicles is often encountered. A benign follicle is composed of a germinal centre surrounded by a rim of dark staining mantle cells which is usually lacking in neoplastic follicles or if present is thin and incomplete [13]. Recognition of neoplastic follicle is histogenetically and prognostically relevant as $\mathrm{FL}$ is a proliferation of $\mathrm{B}$ cells and is an indolent disease and so in the majority, it is not curable. The main problem encountered in routine practice is differentiating follicular hyperplasia from FL. The normal sinus structure of the lymph node is usually identifiable in reactive follicular hyperplasia and is absent in FL. In our study, the provisional diagnoses of the lymph node biopsies were NHL and low grade lymphoma in majority which were eventually diagnosed as FL after IHC. Grading of FL is controversial. Accurate grading in $\mathrm{FL}$ requires well-fixed, well-processed sections. Thick, poorly fixed and overstained sections may lead to of wrong grading of FL. There are clinical and biological differences between grade $3 \mathrm{~A}$ and $3 \mathrm{~B} \mathrm{FL}$. In our study out of 19 cases of grade $3 \mathrm{FL}, 6$ cases were grade $3 B(31.6 \%)$. While FL grade $3 A$ is often considered as an indolent disease and grouped with grades 1-2, data suggest that FL grade 3B has clinical behavior similar to DLBCL so is approached as an aggressive lymphoma [14]. Suspected follicular lymphoma, grade $3 b$ (FL 3b) showing a prominent follicular pattern and with infiltrate entirely composed of centroblasts (absence of centrocytes) were confirmed with CD20 (positive), BCL6 (positive), BCL2 (positive), IRF4/MUM1 (positive) and cyclin D1 (negative). In follicular lymphoma with a follicular pattern (irrespective of grade), CD21 immunostain was used only when the nodular pattern was subtle or was only partially identified. If CD21 demonstrated absence of follicular dendritic cell meshworks, such areas were evaluated for (diffuse large B-cell lymphoma) DLBCL. In FL 3b, BCL2 could be negative. In such cases, strong expression of MUM1 is often helpful. A subset of FLs negative for CD10 is positive for MUM1. Such tumours typically lack the BCL2 translocation but show amplification of BCL6 [4].

Ki67 is a valuable IHC marker to distinguish indolent from aggressive lymphomas especially in small needle biopsies [15]. In our study, the mean proliferative index (Ki-67) among all grades of FL was $34.9 \%$ which is similar to Shih-Sung Chuang, et al. [9]. Xin He, et al. had done a meta-analysis of $\mathrm{Ki}-67$ index in lymphomas and found higher proliferation to be associated with inferior overall survival and disease-free survival rate [16]. Most patients with grades 1-2 have a Ki-67 proliferative index $<20 \%$ [17]. But in our study, it was above $20 \%$ in the cases. Ki67 was correlated with Grade, FLIPI score, stage, number of involved nodal areas, Hemoglobin, bone marrow involvement and peripheral blood involvement. It showed a significant correlation in case of grade, stage, FLIPI score, number of lymph node areas and bone marrow involvement $(P<0.05)$. However, there was no significant correlation in other parameters $(P>0.05)$. Wang, et al. [18] had also proved a high proliferative index as a poor prognostic marker in FL.

Histological transformation of FL to aggressive variants most commonly DLBCL and rarely Burkitt or lymphoblastic lymphoma carries a poor prognosis. In our study, there were three cases of FL showing transformation to DLBCL. The current gold standard definition of transformation is the diffuse effacement of follicular architecture by an increase in large cells, histologically proven and clonally confirmed. It is important to differentiate DLBCL from FL with diffuse architecture and grade $3 \mathrm{~b}$ or IIIb FL. Follicular Lymphoma with diffuse structure shows a mixture of centrocytes and centroblasts and lacks sheets of large cells. FL grade $3 \mathrm{~b}$ has solid sheets of centroblasts ( $>15$ centroblasts $/ 0.159 \mathrm{~mm}^{2}$ ) whereas DLBCL with FL component has a diffuse area with solid sheets of centroblasts outside histologically or immunophenotypically (CD21, CD23 + FDC) recognizable follicles [7].

In our study, there was a case of synchronous presentation of ovarian serous adenocarcinoma and FL in pelvic nodes (Figure 3). Fifty-five year-old postmenopausal woman presented at our institute with complaints of decreased appetite and axillary swelling for 5 months and abdominal distention for 2 months. Her routine investigation including complete blood count $(\mathrm{CBC})$, renal function test, liver function test and coagulation profile were normal except LDH which was 1182 IU/L. Her CT scan of the neck, thorax, abdomen, pelvis was done and was suggestive of bilateral axillary lymph node (largest $14 \times 11 \mathrm{~mm}$ ), para-aortic lymph node (33 $\times 27 \mathrm{~mm})$, pelvic lymph node $(13 \times 12 \mathrm{~mm})$ with hepatomegaly, left-sided mild pleural effusion and gross ascites. Excision biopsy of axillary lymph node was suggestive of low-grade NHL. Her ascitic fluid cytology was suggestive of metastatic adenocarcinoma. Three out of seven pelvic nodes showed FL with metastatic serous adenocarcinoma on morphology. When IHC was done from lymph node it showed positivity for CD20, CD10, $\mathrm{BCl}-2$ and $\mathrm{BCl}-6, \mathrm{CK} 7, \mathrm{WT} 1, \mathrm{PAX}-8, \mathrm{CA} 125$, ER, with negative CD5, CD23 and Cyclin D1, PR, CDX2 and CK20 expression which suggested diagnosis of grade I Follicular lymphoma with metastatic high grade serous adenocarcinoma. Her FLIPI score was 4 (high risk). Collision tu- 
mour of ovarian adenocarcinoma and $\mathrm{FL}$ in lymph nodes is an extremely rare condition. Only a few cases have been reported in the literature [19].

A Bone marrow biopsy is typically performed as part of staging. But many times, clinicians send bone marrow aspiration and biopsies in suspected cases of lymphoma before performing tissue biopsies. Discrepancies sometimes arise between bone marrow and lymph node diagnosis. According to a study conducted by Kwoun, et al. $3 / 66$ cases $(4.5 \%)$ showed discordant results [20]. In the present study, there was a single case where bone marrow morphological diagnosis was lymphoblastic lymphoma but lymph node diagnosis was FL. Immunophenotyping was done which showed $86 \%$ lymphocytes showing expression of CD200, CD10, CD79b, CD20, Lamda and was diagnosed as FL. Flow cytometric detection of $\mathrm{CD} 10+\mathrm{B}$ cell clone is supportive evidence of $\mathrm{FL}$ but because of sampling variability can have negative results. Further as with IHC, the frequency of CD10 expression in bone marrow and blood is decreased. Bone marrow involvement in our study was $30.4 \%$. Eight cases were missed in bone marrow aspiration which was diagnosed in bone marrow biopsy which reinforces the need for bone marrow biopsy for staging. Bone marrow involvement is included in the FLIPI 2 scoring system. Bone marrow involvement was more common in grade 1 and 2 rather than grade 3 cases. Hence bone marrow involvement appears early which is similar to the study by Sylvia, et al. [7] Although currently the FLIPI 2 score [21] is used, we were unable to apply this score in our cases due to incomplete availability of some clinical and biological parameters, which were not routinely collected at the time of diagnosis. These included the size of the largest tumour mass and serum beta- 2 micro globulin level.

Peripheral blood involvement by $\mathrm{FL}$ was seen in 7 cases $(10.14 \%)$ which is lower compared to other studies [7]. Few circulating lymphoma cells do not affect the prognosis, but leukemic spread and high total WBC counts have an adverse prognosis. In our study $2 / 7$ (28.6\%) cases showing lymphoma spillover had a total count of more than 1 lakh. All of these cases were confirmed by immunophenotyping. So multiparameter flow cytometric immunophenotyping has become a powerful tool for diagnosis of B-cell lymphoproliferative disorders by its high throughput and low turnaround time.

\section{Conclusion}

This study highlights the various clinical, pathological and histopathological features of FL. Features like grade, stage, the impact of age, nodal areas, bone marrow and peripheral blood involvement by FL were highlighted. Ki 67 showed a significant correlation in case of grade, stage, FLIPI score, number of lymph node areas and bone marrow involvement. The importance of FLIPI scoring was studied in identifying high-risk cases. There was a significant correlation of LDH with FLIPI score and risk groups in our study. Bone marrow involvement appears early and more common in grade 1 and 2 than grade 3. Leukemic spread and high total WBC counts have an adverse prognosis. Follicular Lymphoma although an indolent disease, risk stratification might help in identifying patients who need treatment initiation and those with less favourable prognosis.

\section{Financial Support and Sponsorship}

Nil.

\section{Conflicts of Interest}

There are no conflicts of interest.

\section{References}

1. Feugier P, Filliatre-Clement $L$ (2019) Recent advances in the first-line treatment of follicular non-Hodgkin lymphoma F1000Res 8: (F1000 Faculty Rev)-294.

2. Roulland S, Kelly RS, Morgado E, Sungalee S, Solal-Celigny $P$, et al. (2014) $t(14 ; 18$ translocation: A predictive blood biomarker for follicular lymphoma. J Clin Oncol 32: 13471355.

3. Swerdlow SH, Campo E, Pileri SA, Lee Harris N, Stein H, et al. (2016) The 2016 revision of the World Health Organization classification of lymphoid neoplasms. Blood 127: 2375-2390.

4. Harris NL, Swerdlow HS, Jaffe ES, Ott G, Nathwani BN, et al. (2008) Follicular lymphoma. In: Swerdlow HS, Campo E, Harris NL, Jaffe ES, Pileri SA, Stien H, Thiele J, Vardiman JW, World Health Organization Classification of Tumours Pathology and Genetics of Tumours of Haematopoietic and Lymphoid Tissues. IARC Press, Lyon, France, 220-226.

5. Carbone A, Roulland S, Gloghini A, Younes A, von Keudell G, et al. (2019) Follicular lymphoma. Nature Reviews Disease Primers 5.

6. Gogia A, Raina V, Kumar L, Sharma A, Sharma MC, et al. (2017) Follicular lymphoma: An institutional analysis. Asian Pacific J Cancer Prev. Asian Pacific Organization for Cancer Prevention 18: 681-685.

7. Sylvia MT, Dey B, Basu D, Jacob SE, Kar R, et al. (2016) Follicular lymphoma: A clinicopathological analysis from a tertiary care institute in southern India. Mediterr J Hematol Infect Dis 8: e2016060.

8. Duarte ÍX, Domeny-Duarte P, Wludarski SCL, Natkunam Y, Bacchi CE (2013) Follicular lymphoma in young adults: A clinicopathological and molecular study of 200 patients. Mod Pathol 26: 1183-1196.

9. Shih-Sung Chuang, Pin-Pen Hsieh, Chin-Li Lu, Yin-Hsuan Wang, Ting-Ying Fu, et al. (2006) A clinicopathologic and molecular study of follicular lymphoma in Taiwan. Clinical Lymphoma Myeloma Leukemia 6: 314-318.

10. Relander T, Johnson NA, Farinha $P$, Connors JM, Sehn $\mathrm{LH}$, et al. (2010) Prognostic factors in follicular lymphoma. Clin Oncol 28: 2902-2913.

11. Alig S, Jurinovic V, Pastore A, Haebe S, Schmidt C, et al. (2019) Impact of age on clinical risk scores in follicular lymphoma. Blood Adv 3: 1033-1038.

12. William BM, Bongu NR, Bast M, Bociek RG, Bierman PJ, et al. (2013) The utility of lactate dehydrogenase in the follow up of patients with diffuse large B-cell lymphoma. Rev Bras Hematol Hemoter 35: 189-191. 
13. Leong AS-Y (2010) Nodular Lymphoid Infiltrates. In: A Pattern Approach to Lymph Node Diagnosis. 83-173.

14. Mustafa Ali M, Rouphail B, Dean RM, Hill BT, Jagadeesh D, et al. (2015) Grade 3 Follicular Lymphoma: Outcomes in the Rituximab Era. Blood 126: 1520.

15. Hashmi AA, Hussain ZF, Faridi N, Khurshid A (2014) Distribution of Ki67 proliferative indices among who subtypes of non-hodgkin's lymphoma: Association with other clinical parameters. Asian Pac J Cancer Prev 15: 8759-8763.

16. He X, Chen Z, Fu T, Jin X, Yu T, et al. (2014) Ki-67 is a valuable prognostic predictor of lymphoma but its utility varies in lymphoma subtypes: Evidence from a systematic meta-analysis. BMC Cancer 14: 153.

17. Dada R (2019) Diagnosis and management of follicular lymphoma: A comprehensive review. Eur J Haematol 103: 152-163.
18. Wang SA, Wang L, Hochberg EP, Muzikansky A, Harris $\mathrm{NL}$, et al. (2005) Low histologic grade follicular lymphoma with high proliferation index: Morphologic and clinical features. Am J Surg Pathol 29: 1490-1496.

19. García J, Campos J, Abdulkader I, León L, López R (2009) [Collision tumor consisting in a metastatic adenocarcinoma and follicular lymphoma in the same lymph node. Report of one case]. Rev Med Chil 137: 83-87.

20. Kwoun WJ, Ahn JY, Park PW, Seo YH, Kim KH, et al. (2019) How useful is bone marrow study as an initial investigative tool without lymph node biopsy in malignant lymphoma?: Eleven years of experience at a single institution. J Clin Lab Anal 33: e22841.

21. Lindsey $H$ (2009) Evaluating FLIPI-2, the new follicular lymphoma prognostic index. Oncol Times 31: 20-22. 